\title{
RESCATANDO EL AUTOCUIDADO DE LA SALUD DURANTE EL EMBARAZO, EL PARTO Y AL RECIÉN NACIDO: REPRESENTACIONES SOCIALES DE MUJERES DE UNA COMUNIDAD NATIVA EN PERÚ ${ }^{1}$ RESCUING THE SELF-CARE OF HEALTH DURING PREGNANCY, THE DELIVERY AND TO THE NEWBORN: SOCIAL REPRESENTATIONS FROM WOMEN OF A NATIVE COMMUNITY IN PERU RESGATANDO O AUTOCUIDADO DA SAÚDE DURANTE A GRAVIDEZ, O PARTO E AO RECÉM-NASCIDO: REPRESENTAÇÕES SOCIAIS DE MULHERES DE UMA COMUNIDADE NATIVA NO PERU
}

\author{
Rocio Elizabeth Chávez. Alvarež, Maria Josefa Arcaya Moncada ${ }^{3}$, Gladys Garcia Arias', Teresa Catalina Surca Rojas5, \\ Maria Virginia Infante Contreras ${ }^{3}$
}

${ }^{1}$ Ganador del Concurso de Proyectos de Investigación, do Consejo Superior de Investigación (CSI) en 2006. Financiado por el
CSI de la Universidad Nacional Mayor de San Marcos (UNMSM).
${ }^{2}$ Magíster en Enfermería Pediátrica. Maestría en Enfermería. Docente Visitante de la UNMSM. Lima, Peru.
${ }^{3}$ Magíster en Enfermería. Docente Principal de lo Departamento de Enfermería de la UNMSM. Lima, Peru.
${ }^{4}$ Licenciada en Enfermería. Docente Auxiliar de lo Departamento de Enfermería de la UNMSM. Lima, Peru.
${ }^{5}$ Magíster en Enfermería. Docente Asociada de lo Departamento de Enfermería de la UNMSM. Lima, Peru.

PALABRAS ClAVE: Autocuidado. Salud maternoinfantil. Población indígena. Conocimientos, actitudes y práctica en salud.

KEYWORDS: Self care. Maternal and child health. Indigenous population. Health knowledge, attitudes, practice.

PALAVRAS-CHAVE: Autocuidado. Saúde maternoinfantil. População indígena. Conhecimentos, atitudes e prática em saúde.
RESUMEN: Este estudio cualitativo tuvo el objetivo de conocer el autocuidado tradicional de mujeres nativas durante el embarazo, el parto y al recién nacido. Fueron efectuadas 16 entrevistas parcialmente estructuradas a madres y parteras de una comunidad nativa peruana. Con el soporte de la teoría de las representaciones sociales y el método etnográfico, emergieron tres categorías temáticas: los símbolos y prácticas tradicionales representadas al cuidarse, preparan a la gestante para un parto fácil; la partera, saberes, prácticas y objetos durante el parto, representan protección para el bebé; la partera y la madre aseguran los cuidados inmediatos y la alimentación del recién nacido. Finalmente, las prácticas de autocuidado tradicional se consideran de sentido común, transmitidas generacionalmente y destacando la mujer cuidadora. Se objetiva respeto por su cosmovisión y naturaleza; y el anclaje de auto cuidado está arraigado a sus costumbres, creencias, símbolos e imágenes, con poca integración del cuidado y salud occidental.

ABSTRACT: The purpose of this qualitative study was to better understand traditional self care about pregnancy, delivery, and for the newborn among native women. For data collection, 16 semi-structured interviews with mothers and midwives of a Peruvian native community were carried out. Upon the support of the social representations theory and ethnographic method, three thematic categories were identified: the symbols and traditional practices represented in applying self-care; preparing the pregnant woman for an easy delivery; the midwife, knowledge, practices, and things during the delivery that represent protection for the baby; and the midwife and the mother assure immediate care and feeding of the newborn. Finally, the practices of traditional self care are considered to be common sense, transmitted by generations, highlighting the woman caregiver. Respect for their cosmo-vision and nature was observed; and the self care anchor is rooted in their customs, beliefs, symbols, and images, with a little integration of occidental care and health.

RESUMO: Este estudo qualitativo teve o objetivo de conhecer o autocuidado tradicional de mulheres nativas durante a gravidez, parto e com o recém-nascido. Foram realizadas 16 entrevistas semi-estruturadas com mães e parteiras de uma comunidade nativa peruana. Com o suporte da teoria das representações sociais e o método etnográfico, emergiram três categorias temáticas: os símbolos e práticas tradicionais representadas no cuidar-se, preparam a gestante para um parto fácil; a parteira, saberes, práticas e objetos durante o parto representam proteção para o bebê; a parteira e a mãe garantem os cuidados imediatos e alimentação do recém-nascido. Finalmente, as práticas tradicionais de autocuidado são consideradas de senso comum, transmitidas de geração em geração e destacam a mulher cuidadora. Objetiva-se o respeito por sua cosmovisão e natureza, e a âncora do autocuidado está arraigada em seus costumes, crenças, símbolos e imagens, mas com pouca integração ao cuidado e saúde ocidental.
Rocío Elizabeth Chávez Alvarez

Dirección: Calle Angélica Palma, 265

CEP: Lima-039 - Los Olivos, Lima, Perú.

Email: jeromiangis@yahoo.com

chioliz@ig.com.br
Artigo original: Pesquisa Recebido em: 16 de abril de 2007 Aprovação final: 25 de outubro de 2007 


\section{INTRODUCCIÓN}

Expresiones culturalmente distintas surgen al tratar de definir el cuidado de la salud en poblaciones multiculturales y contextos también pluriétnicos. Por consiguiente la expresión de culturas diferentes con comportamientos diferentes converge necesariamente en el autocuidado y los cuidados transculturales que de ellas se extrapolan. ${ }^{1}$

Las representaciones sociales ocupan un lugar importante como instrumento explicativo de hechos y fenómenos ante la ausencia de explicaciones científicas y, desde un pensamiento lógico configurado se interpretan los fenómenos naturales y sociales, aunque no correspondan a verdades científicas, como sería el caso de las concepciones diferentes sobre el cuidado de la salud que conciben los pueblos indígenas.

La concepción de salud que tienen los pueblos indígenas, representa una convivencia armónica del ser humano con la natureza, consigo mismo y con los demás hacia un bienestar integral. Lo que refleja su equilibrio entre lo individual (físico, mental, espiritual y emocional) y colectivo (político, económico, cultural y social). Es decir un significado diferente dependiendo del contexto en que es determinado. ${ }^{2-3}$

Es en el mundo simbólico, que el fenómeno de la salud y enfermedad se presenta bajo un marco de referencia codificado, ${ }^{1}$ dando lugar a prácticas y comportamientos específicos con un trasfondo explicativo de difícil transformación, ya que se apoyan en sus mitos, creencias y costumbres arraigadas.

La condición de género surge en estas situaciones, dando a la mujer y al hombre connotaciones diferentes con respecto al cuidado humano, siendo que los cuidados relacionados con el orden público y la aplicación de fuerza física son asignados al hombre, mientras que los cuidados relacionados con todo lo que genera vida, es fecundable y da a luz se revierten en la mujer.

Principalmente los cuidados que tienen que ver con la vida diaria, los alimentos, el cuerpo sano, se acomodan al rol de la cuidadora mujer, ${ }^{4}$ como figura simbólica del eterno femenino en armonía con la naturaleza. Es ella quien se encarga del hogar y su propio cuidado, lo que le permite aprender de su propia experiencia, iniciarse en el conocimiento empírico en su hábitat y proveerse autocuidado de forma innata, práctica que realiza para mantener su propia vida, salud y bienestar basado en los conocimientos que posee. ${ }^{5}$
Los cuidados son esenciales para el bienestar, la salud, la curación, el desarrollo y la supervivencia de una cultura, así como también los conceptos, significados, expresiones, patrones, procesos y formas culturales de los cuidados presentan divergencias y semejanzas en toda cultura, lo que Leininger llama de diversidad y universalidad de los cuidados. ${ }^{1}$

Una propuesta multicultural en salud, elimina la discriminación étnica o cultural, y respeta la diversidad cultural, ratificando así el principio de equidad, libertad e igualdad entre culturas, como conducta social y modo de vida de las personas de forma dinámica e interactiva. El moverse entre estos conocimientos, creencias, prácticas culturales diferentes respecto a la salud y enfermedad, la vida y la muerte, el cuerpo biológico, lo social y relacional; permite pensar en la interculturalidad en salud. ${ }^{6}$

Buscando convergencias entre el autocuidado y la cultura, se encuentran comportamientos arraigados en creencias y tradiciones culturales, la existencia de paradojas comportamentales, ${ }^{8}$ y la socialización estereotipada del cuidado de acuerdo con el sexo; lo que hace difíciles que las personas puedan reemplazar sus costumbres ancestrales por nuevos conocimientos. Así tenemos que en salud los comportamientos están manejados por conocimientos acumulados y representaciones sociales, de las cuales hacen parte las supersticiones y los mitos construidos alrededor del cuidado de la salud.

El acercamiento con las personas al relacionarnos, valorando la visión que de salud-enfermedad tienen, es decir su cultura, no puede desconocerse, pues ejerce gran influencia en la vida de las personas y la población cuando se trabaja en promoción de la salud. ${ }^{8}$ En tal sentido, la cotidianeidad en su totalidad está matizada por la cultura, y sobre salud se puede definir que concepciones de embarazo, parto, puerperio, crianza del niño y otras entidades nosológicas, se enmarcan en un sello particular impuesta por la cultura de sus grupos sociales.

Ya para los pueblos indígenas, la salud es un estado dinámico de equilibrio interno entre el cuerpo y el espíritu, que depende del estado de armonía interna y externa, con los demás seres humanos, la naturaleza y el cosmos en general.,

Los conocimientos tradicionales se relacionan con la supervivencia, modos de producción, expresiones artísticas y religiosas, los modelos de salud y cualquier creencia o concepto, valor o técnica que se use en la vida cotidiana y coexisten distintamente 
a la ciencia occidental. ${ }^{3}$ En este enfoque tradicional, la medicina también cobra fuerza, siendo utilizada como suplemento de los tratamientos occidentales, y donde se observan a curanderos, shamanes, astrólogos, quirománticos, parteras empíricas, espiritistas, entre otros; ${ }^{10}$ haciendo uso de la medicina tradicional y siendo ampliamente divulgada y permitida dentro de las poblaciones indígenas.

Surge también, cercano a este contexto la medicina popular como técnicas de tratamiento utilizados por gente común, que a su vez son transmitidas generacionalmente y con poco reconocimiento por la medicina occidental, pues parte del saber popular de las personas (remedio de la abuela, té casero) para solucionar problemas diarios de la salud. Siendo las personas destacadas en su uso las parteras, rezadoras, curanderos. ${ }^{10}$

El sustento de la Teoría de las Representaciones Sociales, ${ }^{11}$ definido por un conjunto de conceptos, proposiciones y explicaciones que se originan en la vida cotidiana y en el curso de las comunicaciones interpersonales; permite crear información y familiarizarnos con lo desconocido por los procesos de objetivación y anclaje. Trayendo la versión del senso común de los individuos de una comunidad, por medio de sus mitos, creencias y sistemas de conocimiento tradicional. ${ }^{11}$

La objetivación entonces, permite materializar la palabra, transformando una abstracción en lo concreto, físico y tangible. Mientras que el anclaje integra cognitivamente el objeto representado a un sistema de pensamiento social ya existente, lo que se revela con el discurso de los propios sujetos quienes comparten el mismo grupo social, normas establecidas, condiciones y experiencias.

No por el hecho que los pueblos indígenas enfrentan dificultades, puede afirmarse que dejan su salud a un lado, sino por el contrario, recurren a diversas prácticas para su autocuidado, hecho que las hace merecedoras de rescatar su valiosa contribución para las ciencias de la salud con el fin de promover y difundir dichas prácticas contribuyendo en el fortalecimiento del sistema de salud y el aporte de la medicina tradicional, la promoción de la salud indígena y la salud intercultural, ${ }^{13}$ hacia las nuevas e innovadoras tendencias que existen sobre el cuidado de la salud de los pueblos indígenas.

Garantizar y proponer acciones en salud intercultural que solucionen sus problemas emergentes respetando sus concepciones y métodos tradicionales para el cuidado de su salud, es una obligación que todos los Estados deben asegurar, así como restablecer políticas, prioridades y estrategias en la atención de salud acorde con dichas poblaciones, que se integren al sistema de salud indígena. ${ }^{6,12}$

La persistencia de esquemas biomédicos y etnocentristas, ${ }^{13}$ con poca visión de la realidad de los pueblos indígenas, conlleva a intervenciones de salud poco acordes con las necesidades de los usuarios con diversidad multicultural, y mucho menos se concibe la salud como una prioridad de necesidad integral y humanizada, puesto que no se vive esta problemática desde adentro, siendo difícil integrar los conocimientos occidentales y tradicionales para el beneficio de las poblaciones más vulnerables, ${ }^{8,10,13}$ como los pueblos indígenas.

El sistema de salud actual, aun rígido y arraigado al quehacer occidental, no concibe una alternativa más respetable para direccionarse por la interculturalidad en salud y los cuidados integrales de los pueblos indígenas, que valore el respeto y reconocimiento de sus saberes tradicionales y derechos humanos. ${ }^{12}$ Por tal, se desconoce cuan beneficiosa puede ser una atención y cuidados de salud culturalmente pertinentes, que garanticen eficacia y respeten las costumbres hacia una meta conjunta, mejorar la calidad de vida de la población indígena y sentir plena realización como servidores y usuarios de salud.

Por tales cuestionamientos, la investigación se planteó la siguiente interrogante: como son las representaciones sociales del autocuidado de la salud durante el embarazo, el parto y al recién nacido, de las mujeres de la comunidad nativa SIB*?

Por lo que amerita conocer mejor sus representaciones sociales, para difundirlas y garantizar su valiosa contribución a la sociedad y las ciencias de la salud en la actualidad nacional e internacional. En este sentido, el objetivo del estudio fue conocer y comprender las prácticas de autocuidado de la salud durante el embrazo, el parto y al recién nacido, de las mujeres de la comunidad nativa SIB, bajo la óptica de sus representaciones sociales.

\section{TRAYECTORIA METODOLÓGICA}

La investigación tuvo un abordaje cualitativo, captando y comprendiendo los fenómenos relacionados con el autocuidado de la salud, con el 
soporte de la teoría de las representaciones sociales y el método etnográfico. ${ }^{14}$

Desde la perspectiva etnográfica se describió y reconstruyó los escenarios y grupos culturales; se incorporó creencias, experiencias, actitudes, pensamientos y reflexiones de las mujeres nativas, considerando los mismos términos y significados que las participantes le daban a sus acciones. ${ }^{14}$

La investigación se desarrolló durante los meses de junio a agosto del 2006, en la comunidad nativa SIB que pertenece al pueblo indígena ShipiboConibo, del Departamento de Ucayali, en la selva oriental peruana. Participaron 16 mujeres, entre madres (Ma1, Ma2, Ma3, Ma4, Ma5), gestantes (Ge1, Ge2, Ge3, Ge4), puérperas (Pu1, Pu2, Pu3) y parteras $(\mathrm{Pa} 1, \mathrm{~Pa} 2, \mathrm{~Pa} 3, \mathrm{~Pa} 4)$, quienes reunían los criterios de inclusión y saturación delimitados en la investigación cualitativa.

El trabajo de campo comprendió dos momentos; el primero de contacto inicial con la comunidad, coordinaciones y aceptación de las autoridades comunales, ${ }^{14}$ así como la autorización de las participantes señalada en la carta de consentimiento informado, para participar en la investigación, aceptar la entrevista y permitir la difusión de los resultados, fotos y videos con fines científicos exclusivamente. Dejando constancia escrita en el libro de actas comunal y en audio grabado y guardado sigilosamente por los investigadores. De esta forma se garantizó el anonimato de las participantes, siguiendo rigurosamente todas las normas de ética en investigación reguladas por la Declaración de Helsinki, ${ }^{15}$ y la aprobación previa del proyecto de investigación por la Universidad Nacional Mayor de San Marcos, Resolución Rectoral No 060113091.

El segundo momento, cursó con el ingreso al campo y convivencia en la comunidad por 15 días, resaltando la acogida calurosa de las familias. Donde fueron efectuadas entrevistas semiestructuradas como técnica principal de recolección de datos, a las mujeres participantes. El cuaderno de campo y la observación participante, apoyaron en la codificación de datos personales de las participantes, confrontación y ampliación de los datos obtenidos por las entrevistas. Dando así validez al estudio por medio de la triangulación, lo que aseguró la confiabilidad de los resultados de la investigación.

El análisis de los datos cualitativos se apoyo en el análisis de contenido, dando paso a la lectura y re-lectura de cada entrevista, siendo que todas fueron contrastadas por la observación participante, ya que fuimos testigos presenciales de los cuidados impartidos por las participantes en los momentos de su cotidiano tanto como gestantes, puérperas, parteras y madres; basándose en el método etnográfico. Seguidamente, fueron codificadas y separadas en unidades de significados colectivos, por frases y palabras representativas, los cuales dieron lugar a los pre-temas, de donde finalmente emergieron las tres categorías o temas principales del estudio.

El trabajo de campo fue motivador y el cotidiano nos permitió mayor sensibilidad con la situación que viven los pueblos nativos, como estrategias se ejecutaron intervenciones y acciones específicas de salud programadas y coordinadas en el primer momento, como la campaña gratuita de salud integral que benefició a mas de 100 personas, atenciones de salud individualizada y la entrega de una gigantografía bilingüe sobre prevención de diarreas, por ser ese tema de mayor preocupación entre las familias de la comunidad nativa SIB.

\section{RESULTADOS DEL TRABAJO DE CAMPO}

Las principales temáticas abordadas para el análisis e interpretación de los resultados fueron el autocuidado durante el embarazo, el parto y al recién nacido, siendo que todos ellos estaban íntimamente relacionados por ser parte del cotidiano y la vida de la mujer nativa en la comunidad. Se rescataron tres categorías principales llegando a una aproximación de sus representaciones sociales sobre el autocuidado de la salud. Lo que se detalla a seguir.

\section{Los símbolos y prácticas tradicionales repre- sentadas al cuidarse, preparan a la gestante para un parto fácil}

La auto-confirmación del embarazo determinado por algunas molestias de esta etapa, hacen sentir a la mujer diferente aceptando su condición al principio, para luego pasar a un periodo de calma y rutinas como aprendido y transmitido por otras mujeres de su comunidad, por estar presentes desde los albores del tiempo.

Cuando estaba gestando tenia dolor de cabeza [...] ganas de vomitar [...] no podía caminar lejos [...] cuando cumpli tres meses pasó todo (Ge2). Cuando me enteré [...] me sentía bien triste [...] no tenia ganas de estar paseando por allá [...] pasó después de cuatro meses (Ma4). 
Alvarez REC, Moncada MJA, Arias GG, Rojas TCS, Contreras MVI

Cuidados tradicionales en el embarazo transmitidos por generaciones entre mujeres y parteras, ${ }^{10}$ representan escasa incorporación de la medicina occidental, que es utilizada en la medida de sus necesidades de mayor riesgo, puesto que tienen a su alcance los servicios básicos de salud como una segunda alternativa. ${ }^{13}$

[...] si esta complicado la partera sabe su técnica como lo voltea $[. .$.$] ella lo arregla con su mano [...] (Ma2). Mi$ abuela controla el embarazo, sabe tantear como está puesto el bebé $[\ldots]$ pero de aquí pensamos controlar en la posta [...]. (Ge4). [...] las señoras cuando tiene seis meses de embarazo vienen a preguntarme como están [...] les sobo la barriga para saber [...] (Pa1).

Así, el fin de los cuidados está orientado a salvaguardar que el bebé nazca sano, y llegar a un final feliz. En este sentido no se evidencia el embarazo como una experiencia de vida individual, sino que toma en cuenta un parto y nacimiento del bebé sin riesgos. ${ }^{4}$

Otro aspecto, que relució fue la noción de cuidarse. Lo que se vio reflejada en sus acciones efectivas de cuidado, con el apoyo de las mujeres de su comunidad para asegurar su supervivencia como un hecho cotidiano. ${ }^{5}$

A medida que se constituye la vida de un grupo, nace todo ritual, toda una cultura que programa y determina lo que considera bueno o malo para conservar y asegurar la continuidad de la vida, ${ }^{7}$ como comer, lavarse, levantarse, evacuar, moverse, desplazarse. Cuando estoy embarazada voy a la chacra hasta los siete meses [...] cultivo plátano, no cargo mucho peso porque puede causar problemas con el bebito (Ge1). Es importante que la mujer se cuide durante el embarazo para que el hijo nazca desarrollado (Ma3). [...] acuden a ver a la partera [...] para saber si está bien puesto el bebé [...] (Pa2).

Los alimentos de la gestante, son a predominio de los más comunes y provistos por la naturaleza. Siendo loable la adecuación que hacen de ellos, ya que no existe una gama de alimentos para la selección específica de los más nutritivos, pues otros factores de satisfacción de necesidades cobran mayor fuerza, por lo que se aferran a aquellos tan comunes pero vitales como la yuca, el plátano, el pescado y el arroz. ${ }^{6}$

El cuidado de su alimentación, es vista por ellas mismas como un aspecto de normalidad aunque a veces expresen que deben cuidarse mejor por el hecho de estar embarazadas, se puede apreciar que se organizan y consumen alimentos en razón a lo que saben y tienen a su alcance.
Cuando estaba embarazada seguía comiendo igual, pescado en el desayuno almuerzo y cena $[. .$.$] arroz a veces (Ge3).$ La alimentación en mi embarazo ha cambiado [...] ahora como arroz, pescado [...] la persona embarazada debe comer más y mejor para que crezca bien el bebito (Ge1).

La prioridad de usar las plantas medicinales prevalece ante la medicina científica occidental, ${ }^{10}$ para el beneficio de la gestante y para cuidarse evitando embarazos. El continuar aprendiendo y recibiendo cuidados de las otras mujeres de su comunidad es ampliamente evidente al utilizar plantas medicinales para su propio beneficio y un futuro parto de forma rápida. Una de las evidencias más notables de sentido común fue la utilización de la malva chapeada (cruda y molida), como símbolo de preparación durante el embarazo y confianza para un parto sin complicaciones, ${ }^{11}$ por ser considerada efectiva en el dolor de cabeza, inflamaciones y lavados vaginales. ${ }^{6}$

La mujer nativa siempre ha sido curandera, ha usado plantas e intercambiado secretos prácticos entre su género, ${ }^{5}$ forjando sus saberes por esos contactos y a través del tiempo, ${ }^{10}$ ya que por una parte la medicina científica occidental no ha sabido llegar a estas culturas y saberes tradicionales de una forma armónica.

Métodos tradicionales para evitar el embarazo han sido utilizados ampliamente, ya que la experiencia con los métodos artificiales les han traído ciertos riesgos y problemas; disponiendo a las mujeres a aceptarlos o no, según su elección.

No pienso tener mas hijos [...] después de este bebe me pienso tratar con planta medicinal (Ge4). [...] sangre de grado, resina de plátano y piñón [...] me cuidaba con eso, nada de ampolla, nada de pastillas [...] pues me dan hemorragia (Pu1). [...] tomaba malva chapeada todos los días de mi embarazo [...] para ayudar en el parto [...] y tenga menos dolor (Ma3).

\section{La partera, los saberes, las prácticas, y objetos durante el parto representan protección para el bebé}

La preparación para el parto empieza en el embarazo, a través de un saber tradicional aprendido entre mujeres de la comunidad y apoyada por el esposo. Es preparado lo necesario para el parto y nacimiento de su hijo, como la ropa, colcha, destacando el uso del "mosquitero" como imagen-objeto con contenido simbólico, ${ }^{11}$ que se hace visible para la privacidad de la mujer durante el parto y la dedicación exclusiva al recién nacido, transformándose así algo extraño a nuestros ojos en algo familiar y de respeto colectivo en su cultura. ${ }^{11}$ 
Antes de que nazca el bebito, preparo los pañalcitos [...] desde los ocho meses [...] me ayuda mi esposo (Ge3). Empiezo a alistar las cosas para el parto [...] cuando faltan dos meses, $[\ldots]$ alisto mi colcha, mi mosquitero $[. .$.$] también alisto la ropita$ del bebé (Pu3). Estuve preparando las cosas para el parto como pañales, ropitas y el mosquitero en mi embarazo (Ma1).

El trabajo de parto implica la participación de una mujer especialista y con conocimientos tradicionales, ${ }^{10}$ ya que se realiza siempre en el domicilio de la parturienta. Siendo que la responsabilidad recae en la partera de la comunidad, vista como una imagen fundamental por su amplia experiencia, lo que garantiza el nacimiento del bebé preservando sus conocimientos y practicas aceptados por el grupo social. ${ }^{11}$

Este momento es tomado muchas veces con tranquilidad por la familia, que se moviliza para su éxito. Mientras la partera atiende a la mujer directamente, un familiar de confianza permanece junto a la madre, esperando el momento para ayudar en el parto.

Sin embargo, algunas mujeres consideran que deben dar a luz a solas, ya que la presencia de otras personas dificulta el nacimiento del niño y les causa vergüenza. La presencia del esposo a veces se debe a la fuerza física que ayuda si el parto es difícil.

La partera toca la barriga antes del parto y si el bebé está mal puesto entonces lo endereza para que pueda salir (Ma5). [...] me atiende solo la partera y mi madre está presente en mi parto (Pu3). Al momento del parto se necesita la ayuda del varón si el bebé no quiere salir rápido y la mujer sufre, $[\ldots]$ pero si es tranquilo entonces sólo ayuda la mamá de la mujer (Pa3).

Existe un respeto por el pudor de la mujer, la posición que adopta en el parto y enterrar la placenta o shama, como cuidados que han permanecido desde tiempos antiguos, ya que cubren su cuerpo como señal de vergüenza e identidad con su género. La posición sem.-vertical (cuclillas, sem.-inclinada) que adoptan al momento del parto, les da mayor comodidad y facilidad para expulsar al bebé, a diferencia de la horizontal que les parece incómoda, impersonal y de poco respeto por su cuerpo.

La partera también interviene al cubrir a la madre mientras da a luz, y muchas veces sólo con el tacto hace un trabajo eficiente. De esta manera se mantiene el respeto y genera confianza en este momento tan importante para la mujer.

Otra costumbre aprendida y realizada en su colectivo, ${ }^{11}$ es enterrar la placenta o shama, para evitar su contacto con otros animales o el ambiente, hechos representados de manera espontánea sin mucha explicación, pero seguidos firmemente como una costumbre que se mantiene desde mucho tiempo atrás en la comunidad. ${ }^{11}$

Mi mamá me enseñó a dar a luz, arrodillada y en esa posición lo be hecho (Ma1). He atendido mi parto sola porque me da vergüenza y no puedo dar a luz si otros me ven [...] (Pu1). [...] luego enterraron la placenta por que es una costumbre nuestra [...] pornada más (E3). Espero que salga la shama [...] cuando nace le cabo tierra y abi se le mete y se le tapa [...] (Pa1).

La utilización de plantas medicinales es frecuente durante el parto, con la única finalidad de producir menos sufrimiento y dolor a la mujer y facilitar la expulsión del bebé. Hecho también muy respetado en su entorno por los resultados positivos que se pueden apreciar al momento del parto. ${ }^{6,12}$

Cuando el dolor es más fuerte ya sé que va a nacer el bebéy le doy a la mujer hojas de algodón cocinado con malva [...].(Pa2). [...] la familia tiene que cocinar hojas de mango caídas [...] para que ella tome con aceite $[. .$.$] eso ayuda a que resbale rápido el bebé$ (Pa1). [...] entonces tomo boja de piñón y de algodón cocinado para que nazca rápido [...] eso me da la partera (Ma5).

\section{La partera y la madre aseguran los cuidados inmediatos y alimentación del recién nacido}

La partera es un símbolo de identidad colectivo, recepciona y brinda cuidados al recién nacido a través de prácticas tradicionales y objetos caseros que utiliza, para evitar riesgos en el bebé. Se evidencia un gran respeto por su labor en la comunidad, ${ }^{11}$ y es reconocida por brindar confianza y seguridad a la madre.

Es decisión de la madre que la partera la atienda, objetivándose la materialización de objetos, ${ }^{11}$ que son indispensables para garantizar un parto óptimo; como las tijeras hervidas que sirven para cortar el pupo o cordón umbilical, los hilos gruesos de algodón para atar el muñón, y algunas sustancias de plantas medicinales que se aplican en el ombligo del bebé para evitar su infección. El cuidado que brinda la partera responde a sus conocimientos ancestrales, aprendidos y compartidos entre las mujeres dedicadas a esa actividad, seguido de la preservación del estado saludable del recién nacido.

El baño del bebé es otro de los momentos respetados en los cuidados, llevándose a cabo casi después del primer día y dentro del mosquitero, para evitar demasiada luz o males de aire que lo enfermen.,12 Así los comportamientos de estas mujeres en el grupo social, están fuertemente arraigados a su sistema de creencias y tradiciones culturales, involucrando contenidos mágico- 
religiosos, ${ }^{3}$ difíciles de reemplazar por nuevos conocimientos, como objetivado al no exponer al bebé fuera del mosquitero.

Cuando el bebé nace, la partera calentaba agua y tijeras para cortar su pupo (Ge4). Al día siguiente que nace lo baño, [...] le curo el ombligo con resina de piñón blanco por dos días [...] para que lo cure [...] lo seca [...] (Pu2). [...] el bebé no sale hasta los siete días para que no le de mal de aire y no se enferme [...] (Ma4).

El rol de la madre, tiene un sentido afectivo y cariñoso, lo que se observa en los momentos de la lactancia materna y permanencia inmediata con el hijo apenas nace. La lactancia, no es un problema, pues la tienen controlada con la ingesta de masato (jugo de yuca hervida) caliente para aumentar la producción de leche materna. Este comportamiento obedece a un instinto natural de la madre hacia su hijo, integrándose en el rol de cuidadora que la especie humana ha ido formando desde los más remotos tiempos. ${ }^{7}$

La mamá una vez que dio a luz descansa dentro del mosquitero con su bebe, $[\ldots]$ el bebe en el momento que esta naciendo recibe pecho (Pa4). Cuando nace el bebé lo coloco a su lado y le da de mamar (Pa1). Cuando nació en la noche no tomó pechito por que no tenía nada todavía, [...] tomé masato de yuca calientito, bien calientito, eso da leche (Ma2). Cuando recién nació a cada rato mamaba, [...] apenas nació le di pecho (Ma3).

\section{CONSIDERACIONES FINALES}

La perspectiva intercultural de esta investigación permitió identificar problemas del contexto social para una nueva visión que plantee retos en los profesionales de la salud a fin de brindar servicios articulados, culturalmente pertinentes y respetando el saber popular. ${ }^{1,9}$ Es así que la etnografía como método unido a la teoría de las representaciones sociales, permitieron la construcción de los saberes y prácticas de autocuidado de la salud de la mujer en su contexto social y cultural. Los mitos y sistemas de creencias presentes, son vistos como la versión contemporánea del sentido común dando origen a formas de conocimiento socialmente elaboradas y compartidas por las mujeres de la comunidad nativa que constituyen su medio ambiente simbólico. ${ }^{10-11}$

Se aprendió un poco más sobre el sentir, pensar y actuar de los pueblos nativos de la amazonia peruana, en lo que respecta al autocuidado de su salud, con énfasis en la cuidadora principal por excelencia, ${ }^{4}$ la mujer Shipibo-conibo, que prevalece a través de los tiempos.
El uso de plantas medicinales para mejorar las molestias del parto y la utilización de materiales rústicos y transmitidos por las mujeres más antiguas de las familias en la preparación del embarazo, parto y cuidados inmediatos del recién nacido, se han hecho evidentes a lo largo de los discursos colectivos, demostrando gran respeto por su cosmovisión y naturaleza, ${ }^{11}$ de donde obtienen los insumos, aprendiendo y conservando sus prácticas tradicionales hasta la actualidad. ${ }^{12}$

La representación de la mujer partera, ${ }^{10}$ es un símbolo de servicio, por lo que goza de gran reconocimiento y mérito por parte de la comunidad, siendo la principal artífice de los momentos trascendentales durante el embarazo y parto, además de cumplir con los cuidados inmediatos al recién nacido de forma tradicional.

La presencia de la medicina occidental, es aceptada de forma limitada y solo si la mujer presenta algún riesgo durante los procesos del embarazo o parto, o en otros momentos de su periodo de vida, ya que es fundamental en la mujer Shipibo-Conibo conservar su pudor durante la gestación y el parto, lo que es comprendido y respetado por la partera y la colectividad. Esta concepción también debe ser comprendida y respetada por los profesionales de salud, para mejorar la práctica que la medicina occidental impone al descubrir, exponer y manipular excesivamente el cuerpo de la mujer en estas situaciones, ya que puede ser interpretado como agresivas contra la mujer indígena, especialmente durante la atención institucional del embarazo y parto. Y modificadas tomando como ejemplo los cuidados más humanos, sensibles y cálidos que son representados por las comunidades nativas y sus mujeres.

La mujer Shipibo-Conibo cuida su embarazo orientándolo fundamentalmente a salvaguardar el nacimiento de su bebé sano y feliz lo que se evidencia en la objetivación del vínculo afectivo madre-niño durante el nacimiento y los primeros días de su cuidado.

Se pudo evidenciar sigilo y reserva sobre la información de algunas plantas medicinales para su autocuidado (piripiris), manteniendo así un aspecto de su cultura con pocas posibilidades de alteración o fusión con la cultura occidental Hecho que se constituye como un conocimiento de senso común. ${ }^{11}$

Se hace necesaria la adopción de la teoría de las representaciones sociales como referencial den- 
tro de las investigaciones en el campo de salud y enfermería por ser escasa en la producción científica de nuestro medio, dentro de una proposición de cambio de interpretación de la realidad cotidiana en su contexto donde se dan las interrelaciones sujeto-objeto-sujeto.

Debemos reforzar mayor sensibilización pero que vaya de la mano con la acción-intervención de los profesionales de la salud que interactúan con comunidades nativas para el mejor desarrollo intercultural y calidad de vida garantizada colectivamente, ya que se observa todavía escasa integración de los cuidados y salud occidental.

Finalmente, desde nuestro pensar y sentir como enfermeras, consideramos que la etnoenfermeria peruana presenta un rico potencial para su desarrollo, ${ }^{1}$ si se conoce y aprende de la heterogeneidad cultural de la población y la necesidad de encontrar nuevos caminos que contribuyan desde la academia y la práctica de Enfermería a luchar contra la desigualdad y la falta de equidad de los servicios de salud en poblaciones excluidas socialmente como la comunidades nativas y andinas del Perú. ${ }^{2}$

\section{REFERENCIAS}

1 Leininger M. Transcultural nursing: concepts, theories, research and practice. In: Leininger $M$, McFarland MR, editors. Transcultural nursing: concepts, theories, research and culture. 3rd ed. Toronto (ON/CA): McGraw-Hill; 2002. p.77-98.

2 Organización Mundial de la Salud, Organización Panamericana de la Salud. Documento CD37/20 sobre la iniciativa Salud de los Pueblos Indígenas de las Américas [on line]. Washington (DC/USA): OPS; 1993 [citado 2006 Feb 1]. Disponible en: http://www. paho.org/spanish/ad/ths/os/indig-Resol-V.htm

3 Flores-Guerrero R. Salud, enfermedad y muerte: lecturas desde la antropología sociocultural. Rev. Mad [serie de Internet] 2004 May; (10): [8p.] [citado 2006 Ago 03]. Disponible en: http://www.revistamad. uchile.cl/10/paper03.pdf
4 Martin VB, Angelo M. A organização familiar para o cuidado dos filhos: percepção das mães em uma comunidade de baixa renda. Rev. Latino-Am. Enferm. 1999 Oct; 7 (4): 89-95.

5 Collière MF. Promover la vida. 1a ed. Madrid (ES): Interamericana; 1993.

6 Ministério de Salud (PE), Dirección Regional de Salud Ucayali. Análisis de la situación de salud del pueblo Shipibo-Conibo de Ucayali-ASIS. Pucallpa (PE): Dirección Regional de Salud Ucayali; 2002.

7 De-Roux G. La prevención de comportamientos de riesgo y la promoción de estilos de vida saludables en el desarrollo de la salud. Educación Médica y Salud. 1994 Abr; 28 (2): 223-33.

8 Monreal LA, Maya AP. Compartiendo una experiencia de promoción de la salud en una situación de interculturalidad. México (DF/MX): Instituto Nacional de Salud Pública/Universidad Autónoma de México; 2000.

9 Helman CG. Cultura, saúde \& doença. 4a ed. Porto Alegre (BR): Artmed; 2003. 408p.

10 Siqueira KM, Barbosa MA, Brasil VV, Oliveira LMC, Andraus LMS. Crenças populares referentes à saúde: apropriação de saberes sócio-culturais. Texto Contexto Enferm. 2006; 15 (1): 68-73.

11 Moscovici S. Psicología social II: pensamiento y vida social. Barcelona (ES): Paidós; 1993.

12 Reátegui J. Las políticas de salud de los pueblos indígenas de la amazonia peruana. Rev. Voz Indígena. 2000 Ene-Mar; 1 (1): 4-8.

13 Acosta M, Alegría L, Cajiao GE, Llano AM, Valencia C, Zuluaga P. Creencias populares sobre el autocuidado durante el puerperio en las instituciones de salud de nivel 1. Rev. Colombia Médica. 1997 EneMar; 28 (1): 42-50.

14 Hammersley M, Atkinson P. Etnografía. Buenos Aires (AR): Paidós; 1983.

15 Asamblea Médica Mundial [página de internet]. Declaración de Helsinki: principios éticos para las investigaciones médicas en seres humanos. Tokio: WMA; [actualizado 2007 Set 10; acceso en 2007 Feb 12]. Disponible en: http://www.wma.net/s/ policy/pdf/17c.pdf 